\title{
Prospective Validation of a Decision Tree Model for Prediction of Severity in Acute Pancreatitis
}

Tanka Prasad Bohara, ${ }^{1}$ Uttam Laudari, ${ }^{1}$ Anuj Parajuli, ${ }^{1}$ Shail Rupakheti, ${ }^{1}$ Mukund Raj Joshi ${ }^{1}$

${ }^{1}$ Kathmandu Medical College Teaching Hospital, Sinamangal, Kathmandu, Nepal.

\begin{abstract}
Background: Early identification of severe acute pancreatitis is important for early stratification, goal directed fluid therapy, rationalizing level of care to improve outcome. Various clinical, laboratory and imaging scoring system has been used to identify severe acute pancreatitis with variable results. Recently a decision tree model was proposed using serum creatinine, serum lactate dehydrogenase and oxygenation index to predict severe acute pancreatitis. This system is easy and usable at our centre. Hence, we conducted the study to validate the decision tree model prospectively.

Methods: Patients admitted with a diagnosis of acute pancreatitis were included in the study. Decision tree model was used to identify patients at high and low risk for severe acute pancreatitis. Sensitivity and specificity were calculated for prediction of the decision tree model.

Results: Fifty-three patients were included in the study. Fourty-one $(77.4 \%)$ patients with mild acute pancreatitis, five $(9.4 \%)$ patients had moderately severe pancreatitis and seven $(13.2 \%)$ patients had severe acute pancreatitis. Sensitivity and specificity of decision tree model to predict severity of pancreatitis was $97.83 \%(95 \% \mathrm{CI}-88.47 \%$ to 99.94\%) and $71.43 \% \%(95 \% \mathrm{CI}-29.04 \%$ to $96.33 \%$ ) respectively with positive and negative predictable value of $95.74 \% \%$ (95 \% CI $-87.45 \%$ to $98.64 \%$ ) and $83.33 \% \%(95 \% \mathrm{CI}-40.49 \%$ to $97.35 \%)$ respectively.

Conclusions: Decision tree model with serum creatinine, lactate dehydrogenase, and oxygenation index is an easy and useful tool to predict patients at high risk of developing severe acute pancreatitis.

Keywords: Acute pancreatitis; decision tree; severe acute pancreatitis.
\end{abstract}

\section{INTRODUCTION}

Acute pancreatitis (AP) is an inflammation of pancreatic and peripancreatic tissue. ${ }^{1}$ AP is usually mild and selflimiting. About $25 \%$ of patients develop severe disease with local and systemic complications in whom mortality may reach up to $30 \%$. ${ }^{2-4}$ Early identification of the patient at risk of severe acute pancreatitis(SAP) helps to provide individualized care.

The search for an ideal method for early identification of SAP is continued. Several clinical, biochemical, imaging scores and single parameters have been evaluated with variable results. . $^{2,-10}$

Recently a decision tree model comprising of serum creatinine, serum lactate dehydrogenase and oxygenation index has been proposed in a retrospective study and has suggested prospective and broader validation. ${ }^{11}$ This tool seems useful and feasible to use in our setup. Hence, we conducted a study to validate the decision tree model to predict the severity of AP in a prospective study.

\section{METHODS}

Patients admitted in Department of Surgery Kathmandu Medical College Teaching Hospital from June 2016 to May 2017 with a diagnosis of AP were included in the study. Patients less than 18 years of age, patients with chronic kidney disease, cardiac failure and respiratory dysfunction and patients referred from other centers in whom the serum creatinine, serum lactate dehydrogenase and oxygenation index at admission were not available were excluded.

AP was diagnosed based on revised Atlanta Classification if two of the following three features were present (a) abdominal pain consistent with AP; (b) serum amylase (or lipase) more than three times normal value; (c) characteristic findings of AP on abdominal ultrasound, contrast enhanced computed tomography (CECT) abdomen or magnetic resonance imaging $(\mathrm{MRI}){ }^{12}$

Patients demographic data, history, physical examination findings, biochemical investigations (liver function
DOI: $\underline{\text { http: //dx.doi.org/10.3126/ }}$ inhrc.v16i2.20318 
tests, renal function test) and findings of chest $\mathrm{x}$-ray, abdominal ultrasound, contrast enhanced computerized tomography (CECT) abdomen (if done) and magnetic resonance imaging (MRI) of the abdomen (if done) were noted in a case sheet. Presence or absence of organ failure based on Modified Marshall scoring system for organ dysfunction and systemic inflammatory response syndrome were noted. Arterial blood gas (ABG) analysis was done only when the patients had any sign of respiratory failures, such as the respiratory rate (RR) of more than 26 breaths per minute and the oxygen saturation ( $\mathrm{SpO} 2$ ) detected in the finger less than $90 \%$. Oxygenation index $(\mathrm{Ol})$ was calculated by dividing partial pressure of oxygen in arterial blood with a fraction of inspired oxygen $\left(\mathrm{PaO}_{2} / \mathrm{FiO}_{2}\right)$. For patients without above signs of respiratory failure, $\mathrm{OI}$ was considered more than 289.

Decision tree model proposed by Yang et al was used to identify the high-risk patients for the development of SAP (Fig. 1). Patients were categorized into high risk group when serum creatinine $>196.4 \mu \mathrm{mol} / \mathrm{l}$ or serum creatinine $<196.4 \mu \mathrm{mol} / \mathrm{l}$ and serum $\mathrm{LDH}>536 \mathrm{U} / \mathrm{l}$ or serum creatinine $<196.4 \mu \mathrm{mol} / \mathrm{l}$, serum $\mathrm{LDH}<536 \mathrm{U} / \mathrm{l}$ and $\mathrm{OI}<289$. Patients with serum creatinine $<196.4 \mu \mathrm{mol} / \mathrm{l}$, serum $\mathrm{LDH}<536 \mathrm{U} / \mathrm{l}$ and $\mathrm{Ol}>289$ will be categorized as low risk for development of SAP. These investigations were obtained within 12 hours of admission.

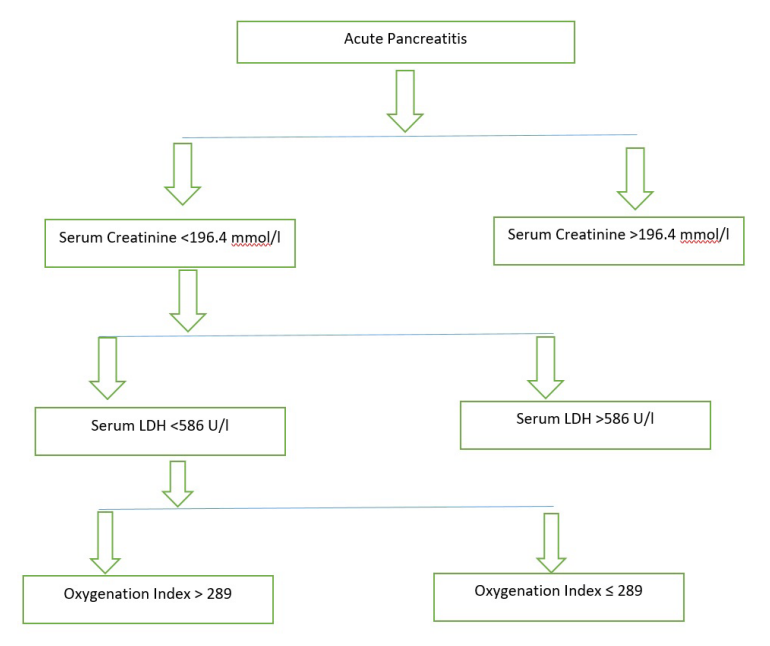

Figure 1. Decision tree model for prediction of Severity of Acute Pancreatitis. ${ }^{11}$

Revised Atlanta Classification criteria were used to classify mild acute pancreatitis (MAP), moderately severe acute pancreatitis (MSAP) and severe acute pancreatitis (SAP). Patients without organ failure and local complications were classified as MAP. Patients with transient organ failure (less than 48 hours) or with local complications were classified as MSAP and patients with organ failure persisting beyond 48 hours were classified as SAP.

Continuous variables were reported as mean \pm SD where as categorical variables were reported as absolute or relative frequencies. Krusal Wallis test was used to analyze continuous variables because of non-parametric distribution of data. Chi square test or Fischer exact test were used for categorical variables whichever was appropriate. $p$ value of $<0.05$ was considered statistically significant. SPSS version 20 was used for analysis of data. The sensitivity, specificity, positive and negative predictive value of the decision tree model to predict development SAP among the high-risk group and predict non-severe pancreatitis in low-risk group was calculated. For calculation of sensitivity and specificity, MAP and MSAP were combined into a nonsevere pancreatitis group and compared with SAP group. Sensitivity and specificity were calculated for prediction of severe and non-severe pancreatitis using two by two tables. Receiver operating characteristics (ROC) curve was plotted for prediction of SAP using decision tree model.

Ethical clearance was taken from the Research and Ethics committee of Kathmandu Medical College Teaching Hospital, Sinamangal, Kathmandu, Nepal. Informed consent was taken from the patients.

\section{RESULTS}

Sixty patients were admitted with a diagnosis of AP during the study period. Seven patients were excluded from the study. Fifty-three patients were enrolled in the study. Mean age was 43.47 years (Range - 18 -79 years). There were 35 males (66\%) and 18 females (34 $\%)$. There were 41 (77.4\%) patients with MAP, five (9.4\%) patients with MSAP and seven (13.2\%) patients with SAP. One patient died within 24 hours of admission and was included in SAP group although persistent organ failure (for $>48$ hours) could not be observed.

Table 1 shows baseline characteristics of the patients in MAP, MSAP and SAP group. The difference was statistically significant in pulse rate at admission, temperature, oxygen saturation, SIRS score, platelet count, direct bilirubin, lactate dehydrogenase, serum amylase and serum urea.

Table 2 shows length of intensive care unit (ICU), surgical high care unit (SHCU) and hospital stay of patients in MAP, MSAP and SAP group. All parameters were more in MSAP and SAP than MAP group and it was statistically significant. 


\begin{tabular}{|c|c|c|c|c|}
\hline \multicolumn{5}{|c|}{ Classification of Severity based on Revised Atlanta Classification } \\
\hline Variables & $\begin{array}{r}\text { Mild Acute } \\
\text { Pancreatitis }\end{array}$ & $\begin{array}{l}\text { Moderately Severe } \\
\text { Acute Pancreatitis }\end{array}$ & $\begin{array}{r}\text { Severe Acute } \\
\text { Pancreatitis }\end{array}$ & P value* \\
\hline Age (in years) & $43.93 \pm 15.115$ & $43.80 \pm 6.017$ & $40.57 \pm 14.386$ & 0.908 \\
\hline $\begin{array}{l}\text { Interval Between onset of } \\
\text { symptoms and admission (in } \\
\text { hours) }\end{array}$ & $47.10 \pm 55.078$ & $79.00 \pm 59.523$ & $66.86 \pm 55.078$ & 0.138 \\
\hline Pulse rate & $90.10 \pm 16.463$ & $109.40 \pm 18.995$ & $120.75 \pm 28.371$ & 0.006 \\
\hline $\begin{array}{l}\text { Systolic blood pressure (in } \mathrm{mm} \\
\text { of } \mathrm{Hg} \text { ) }\end{array}$ & $124.66 \pm 24.058$ & $122.00 \pm 30.332$ & $118.57 \pm 24.785$ & 0.510 \\
\hline $\begin{array}{l}\text { Diastolic blood pressure (in } \\
\mathrm{mm} \text { of } \mathrm{Hg} \text { ) }\end{array}$ & $80.49 \pm 12.031$ & $80.00 \pm 20.000$ & $75.71 \pm 17.182$ & 0.770 \\
\hline Respiratory Rate & $20.34 \pm 2.565$ & $20.00 \pm 2.000$ & $25.14 \pm 8.552$ & 0.118 \\
\hline Temperature (in Fahrenheit) & $98.053 \pm 0.990$ & $99.06 \pm 1.131$ & $98.90 \pm 1.550$ & 0.031 \\
\hline $\begin{array}{l}\text { Oxygen Saturation (in } \\
\text { percentage) }\end{array}$ & $96.20 \pm 4.045$ & $96.40 \pm 1.140$ & $90.43 \pm 6.876$ & 0.012 \\
\hline SIRS Score & $1.20 \pm 0.813$ & $2.00 \pm 0.707$ & $2.43 \pm 0.787$ & 0.002 \\
\hline Haemoglobin (in gram \%) & $17.67 \pm 20.337$ & $12.26 \pm 2.261$ & $14.685 \pm 2.551$ & 0.125 \\
\hline $\begin{array}{l}\text { White Blood Cells Count (per } \\
\text { cu. mm.) }\end{array}$ & $14819.51 \pm 5867.164$ & $15060.00 \pm 5205.574$ & $14028.57 \pm 5579.341$ & 0.800 \\
\hline Platelets (per cu. mm) & $\begin{array}{r}248195.12 \pm \\
82089.652\end{array}$ & $\begin{array}{r}358200.00 \pm \\
202909.004\end{array}$ & $\begin{array}{r}162428.57 \pm \\
36650.862\end{array}$ & 0.014 \\
\hline Random blood sugar (mg/dl) & $133.22 \pm 62.279$ & $122.00 \pm 59.245$ & $125.29 \pm 37.353$ & 0.586 \\
\hline Total Bilirubin (mg/dl) & $2.11 \pm 1.789$ & $1.20 \pm 0.60415$ & $3.53 \pm 3.573$ & 0.140 \\
\hline Direct Bilirubin (mg/dl) & $1.12 \pm 1.123$ & $0.340 \pm 0.260$ & $2.37 \pm 2.774$ & 0.021 \\
\hline Aspartate Transaminase (U/L) & $109.15 \pm 112.965$ & $46.00 \pm 33.496$ & $149.00 \pm 208.207$ & 0.289 \\
\hline Alanine Transaminase (U/L) & $120.27 \pm 115.254$ & $53.80 \pm 37.171$ & $185.14 \pm 103.296$ & 0.299 \\
\hline Alkaline Phosphatase (U/L) & $350.29 \pm 256.923$ & $337.80 \pm 398.825$ & $185.14 \pm 103.296$ & 0.179 \\
\hline Lactate Dehydrogenase (U/L) & $332.51 \pm 112.131$ & $411.40 \pm 171.572$ & $538.86 \pm 184.867$ & 0.015 \\
\hline Serum Amylase (U/L) & $1131.00 \pm 1049.257$ & $259.80 \pm 195.999$ & $2245.43 \pm 4532$ & 0.084 \\
\hline Urea (mg/dl) & $26.7317 \pm 9.162$ & $29.20 \pm 2.774$ & $72.428 \pm 70.604$ & 0.007 \\
\hline Serum Creatinine $(\mathrm{mg} / \mathrm{dl})$ & $0.770 \pm 0.170$ & $0.820 \pm 0.238$ & $1.928 \pm 2.856$ & 0.183 \\
\hline
\end{tabular}

Table 2. Length of ICU, SHCU and hospital stay of patients in MAP, MSAP and SAP group.

Classification of Severity based on Revised Atlanta Classification

\begin{tabular}{lrrrr} 
Variables & $\begin{array}{r}\text { Mild Acute } \\
\text { Pancreatitis }\end{array}$ & $\begin{array}{r}\text { Moderately Severe } \\
\text { Acute Pancreatitis }\end{array}$ & $\begin{array}{r}\text { Severe Acute } \\
\text { Pancreatitis }\end{array}$ & P value \\
\hline Length of ICU stay (in days) & 0 & $1.00 \pm 2.236$ & $5.57 \pm 4.614$ & $<0.001$ \\
Length of SHCU stay (in days) & $1.68 \pm 1.350$ & $5.60 \pm 5.367$ & $4.43 \pm 4.894$ & 0.001 \\
Length of Hospital stay (in days) & $5.44 \pm 3.178$ & $12.20 \pm 11.122$ & $14.86 \pm 6.858$ & $<0.001$ \\
\hline
\end{tabular}

Table 3. Comparison of prediction of severity based on decision tree model and diagnosis based on Revised Atlanta Classification.

Diagnosis based on Revised Atlanta Classification

$\begin{array}{rrr}\begin{array}{l}\text { Prediction based on } \\ \text { decision tree model }\end{array} & \begin{array}{r}\text { Non-Severe Pancreatitis (MAP + MSAP) } \\ \text { Frequency (Percentage within row) }\end{array} & \text { Frequency (Percentage within } \\ & \text { row) }\end{array}$

\begin{tabular}{|c|c|c|c|}
\hline $\begin{array}{l}\text { Non-Severe Pancreatitis } \\
(\text { MAP + MSAP) }\end{array}$ & 45 (95.74 \%) & $2(4.26 \%)$ & $47(100 \%)$ \\
\hline Severe Acute Pancreatitis & 1 (16.67\%) & $5(83.33 \%)$ & $6(100 \%)$ \\
\hline Column Total & 46 & 7 & \\
\hline
\end{tabular}


Most common etiology was biliary, seen in 28 (45.3\%) patients, followed by alcohol in 22 (41.5\%). Three (5.7\%) patients had idiopathic pancreatitis. Among patients with biliary pancreatitis, one patient had a choledochal cyst, one patient had a history of open cholecystectomy and choledochoduodonostmy and two patients had pancreatitis following cholecystectomy.

Based on the decision tree model 47 patients were predicted to be in a low risk group and to have a nonsevere course. Among them, 45(95.74\%) had a nonsevere course. Among six patients predicted as high risk for SAP, five $(83.33 \%$ ) had SAP, as shown in Table 3. Considering decision tree model as diagnostic test, decision tree model had sensitivity of $97.83 \%(95 \% \mathrm{Cl}$ $88.47 \%$ to $99.94 \%)$, specificity of $71.43 \% \%(95 \% \mathrm{Cl}$ $29.04 \%$ to $96.33 \%$ ) with positive and negative predictable value of $95.74 \% \%$ (95\% Cl - $87.45 \%$ to $98.64 \%$ ) and 83.33 $\%$ \% (95 \% Cl - 40.49\% to $97.35 \%$ ) respectively. Figure 2 shows receiver operating characteristic curve (ROC) for prediction of severity of acute pancreatitis based on the decision tree model. The area under the curve was 0.846, $\mathrm{p}$ value 0.003 with $95 \% \mathrm{Cl}-0.641$ to 1.00 .

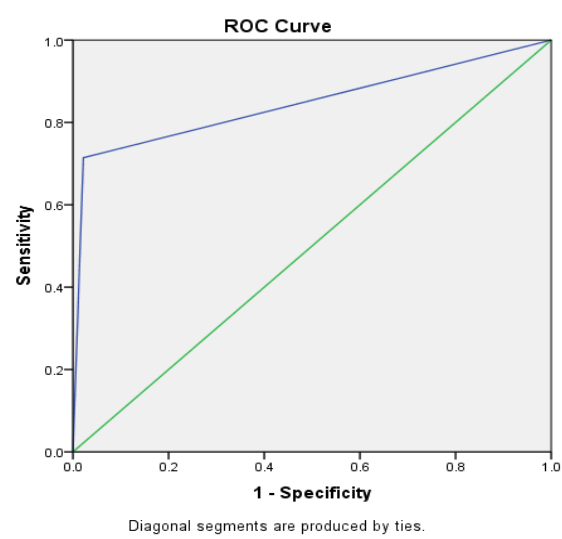

Figure 2. Receiver operating characteristic curve for prediction of severity based on decision tree model.

There was one mortality among the study group. Two patients required endoscopic retrograde cholangiopancreatography(ERCP) and stone clearance in index admission. One patient required ultrasound guided percutaneous intervention. Two patients were readmitted within a month for pain abdomen out of which one belonged to MAP group and other to MSAP group.

\section{DISCUSSION}

AP is an acute inflammation of pancreas with both local and systemic complications. It may present as mild self-limiting disease to a fulminant condition. Patients with a mild form of the disease usually improve in a few days with supportive treatment. However, patients with severe form of the disease are at risk of developing life-threatening complications. In about $25 \%$ of the patient severe form of the disease is seen with local and systemic complications in whom mortality is seen in up to $30 \% .2,3,10$

Revised Atlanta classification has classified AP into MAP, MSAP and SAP. ${ }^{12}$ MAP only requires supportive treatment and observation for few days. MSAP requires goal directed fluid resuscitation, may require fasting, better and close monitoring to see whether the organ failure is transient or persists beyond 48 hours. ${ }^{1}$ SAP is classified if the organ failure persists beyond 48 hours. ${ }^{12}$ However, the patients with SAP are the one who actually requires intensive care, aggressive fluid resuscitation, and close monitoring or transfer to a tertiary care center. Early identification of patients at risk of developing a severe form of the disease is very important. Early identification allows triage of the patients and sorting patients requiring high care and intensive care treatment. Rationalization of the level of care is very important in our context because of limited resources. Early identification allows early aggressive treatment such as goal directed fluid therapy which restores intravascular volume and improves peripheral circulation which prevents tissue hypoxia and further complications. ${ }^{1,13}$ Early identification also allows proper counseling of the patients and their relatives regarding the possible prognosis and hospital course.

The importance of early identification of the patient at risk of severe disease is well known but early identification of the patient at risk of SAP is difficult. Till now no gold standard parameter or scoring system has been developed. A number of researches have been done for decades to identify the simple, fast, reproducible, easily available and cost-effective parameter or a scoring system. There are several clinical, biochemical and imaging systems and scores to predict the severity of AP. Most commonly used methods are Ranson's criteria, Acute Physiology and chronic health evaluation (APACHE II), Bedside index for severity in acute pancreatitis (BISAP), Computerized Tomography (CT) criteria, Japanese severity score..$^{2,5-9}$ Disadvantages of these methods are that the parameters included in these scoring systems are not available at all centers and some take at least 24 to 48 hours for calculation. Single parameters such as haematocrit, C- Reactive Protein, Interleukin, Blood urea nitrogen (BUN) have also been evaluated with varying results. ${ }^{10}$

Recently a simple tool, a decision tree model comprising 
of serum creatinine, serum lactate dehydrogenase and oxygenation index has been proposed by Yang et al (figure 1) in a retrospective study and has suggested prospective and broader validation of this tool. ${ }^{11}$ This tool seems useful and feasible to use at our setup and may be useful in risk stratification of acute pancreatitis patients. "A decision tree is a decision support tool that uses a tree-like graph or model of decisions and their possible consequences, including chance event outcomes, resource costs, and utility". ${ }^{14}$ Yang et al randomized 603 patients into a training group $(n=402)$ and test group $(n=201)$. Revised Atlanta classification was used to classify patients into MAP, MSAP and SAP. Prediction model was constructed using the decision tree method using univariate and multiple regression analysis and diagnostic sensitivity, specificity, and accuracy of individual parameters. The parameters creatinine, LDH, and $\mathrm{OI}$ were selected with the cutoff values were 196.4 $\mu \mathrm{mol} / \mathrm{L}, 536 \mathrm{U} / \mathrm{L}$, and 289 respectively, as shown in figure 1. Developed decision tree model was evaluated in the training group. The sensitivity, specificity, and overall accuracy of the developed model in diagnosing SAP were $80.9 \%, 90.0 \%$, and $88.5 \%$ respectively. The model was again evaluated in the test group $(n=201)$. The sensitivity and specificity of the developed decision model in the test group were $88.6 \%$ and $90.4 \%$ respectively. The probabilities of developing SAP in the high-risk and low-risk groups were $65.9 \%(31 / 47)$ and $2.6 \%$ (4/154), respectively. ${ }^{11}$ The current study used same prediction model developed by Yang et al to predict SAP. Prediction model predicted 47 patients to have non-severe acute pancreatitis, out of which 45 (95.74\%) had a non-severe disease where as two (4.26\%) developed severe disease. Out of six patients predicted to have developed SAP, five (83.3\%) developed SAP and one (16.67\%) developed nonsevere pancreatitis. Sensitivity and specificity of this prediction model in the current study were $97.83 \%$ and $71.43 \%$ respectively with a positive predictable value of $95.74 \%$ and negative predictable value of $83.33 \%$. These results are comparable to Yang et al. Similarly, receiver operator characteristic analysis (ROC) showed the comparable result with Yang et al, the area under curve 0.846 in the current study compared to 0.855 .

Hong et al had also developed a decision tree model using blood urea nitrogen, pleural effusion, and serum calcium as parameters to predict SAP. They tested the model in test group showed that the sensitivity and specificity of their model were $78.9 \%$ and $93.1 \%$ respectively. ${ }^{15}$ They have used original Atlanta classification to classify patients into mild and severe acute pancreatitis. We preferred model developed by Yang et al because they have used latest Revised Atlanta classification and classified patients into MAP, MSAP and SAP.

One patient in this study presented with fulminant acute pancreatitis and died within 24 hours of admission. This patient was included in SAP group though persistent organ failure could not be observed. This signifies the limitation of the current revised Atlanta classification which requires persistent organ failure (for > 48 hours) to classify a patient as SAP. We agree with Huang and Windsor's recommendation for inclusion of this severe form of AP by modification of current classification. ${ }^{16}$ Similarly Thompson has suggested to propose modification of revised Atlanta Classification to include a separate subcategory ("fulminant acute pancreatitis"), to describe cases of SAP, in which death occurs so early that there has not been enough time for the organ failure to have been present for 48 hours or for a day 3 CT scan to have been performed. ${ }^{17}$

This study has certain limitations. This is a single center study with relatively small sample size. This study had a small number of patients with SAP. The results may not be generalized. Hence, a multicentre study with a large number of patients with SAP would be needed to accept the results universally.

\section{CONCLUSIONS}

Decision tree model with serum creatinine, lactate dehydrogenase, and oxygenation index is an easy and useful tool to predict patients at high risk of developing SAP.

\section{ACKNOWLEDGEMENTS}

We would like to acknowledge Dr Manish Pokhrel, Dr Mahipendra Tiwari and Dr Erena Pradhan for their help.

\section{REFERENCES}

1. Talukdar R, Swaroop Vege S. Early management of severe acute pancreatitis. Curr Gastroenterol Rep [Internet]. 2011 Apr 18 [cited 2017 Jun 10];13(2):123-30. [Link]

2. Domínguez-Muñoz JE, Carballo F, García MJ, de Diego JM, Campos R, Yangüela J, et al. Evaluation of the clinical usefulness of APACHE II and SAPS systems in the initial prognostic classification of acute pancreatitis: a multicenter study. Pancreas [Internet]. 1993 Nov [cited 2015 Mar 2];8(6):682-6. [PubMed]

3. Tenner S, Sica G, Hughes M, Noordhoek E, Feng S, Zinner $\mathrm{M}$, et al. Relationship of necrosis to organ failure in severe acute pancreatitis. Gastroenterology [Internet]. 1997 Sep [cited 2015 Mar 3];113(3):899-903. [PubMed] 
4. Sharma M, Banerjee D, Garg PK. Characterization of newer subgroups of fulminant and subfulminant pancreatitis associated with a high early mortality. Am J Gastroenterol [Internet]. 2007;102(12):2688-95. [PubMed]

5. Woo SM, Noh MH, Kim BG, Hsing CTer, Han JS, Ryu SH, et al. Comparison of Serum Procalcitonin with Ranson, APACHE-II, Glasgow and Balthazar CT Severity Index Scores in Predicting Severity of Acute Pancreatitis. Korean J Gastroenterol [Internet]. 2011 Jul [cited 2014 May 6];58(1):31-7. [Full Text]

6. Papachristou GI, Muddana V, Yadav D, O'Connell M, Sanders MK, Slivka A, et al. Comparison of BISAP, Ranson's, APACHE-II, and CTSI Scores in Predicting Organ Failure, Complications, and Mortality in Acute Pancreatitis. Am J Gastroenterol [Internet]. 2010 Feb 27 [cited 2017 Aug 13];105(2):435-41. [PubMed]

7. Ranson JH. Etiological and prognostic factors in human acute pancreatitis: a review. Am J Gastroenterol [Internet]. 1982 Sep [cited 2015 Feb 2];77(9):633-8. [PubMed]

8. Blum T, Maisonneuve P, Lowenfels a B, Lankisch PG, Georg P. Fatal outcome in acute pancreatitis: its occurrence and early prediction. Pancreatology [Internet]. 2001 Jan [cited 2014 Aug 25];1(3):237-41. [DOI]

9. Kuo DC, Rider AC, Estrada P, Kim D, Pillow MT. Acute Pancreatitis: What's the Score? J Emerg Med [Internet]. 2015 Jun [cited 2017 Aug 13];48(6):762-70. [Full Text]

10. Phillip V, Steiner JM, Algül H, Phillip V, Steiner JM, Algül $H$, et al. Early phase of acute pancreatitis : Assessment and management. World J Gastrointest Pathophysiol [Internet]. 2014;5(3):158-68. [PubMed]
11. Yang Z, Dong L, Zhang Y, Yang C, Gou S, Li Y, et al. Prediction of Severe Acute Pancreatitis Using a Decision Tree Model Based on the Revised Atlanta Classification of Acute Pancreatitis. PLoS One [Internet]. 2015;10(11):e0143486. [Full Text]

12. Banks PA, Bollen TL, Dervenis C, Gooszen HG, Johnson CD, Sarr MG, et al. Classification of acute pancreatitis 2012 : revision of the Atlanta classification and definitions by international consensus. Gut [Internet]. 2013 Jan [cited 2014 Apr 29];62(1):102-11. [Full Text]

13. Stigliano S, Sternby H, de Madaria E, Capurso G, Petrov MS, Stigliano AS, et al. Early management of acute pancreatitis: a review of the best evidence. Dig Liver Dis [Internet]. 2017 Jun [cited 2017 Aug 13];49(6):58594. Available from: http://dx.doi.org/10.1016/j. dld.2017.01.168

14. Decision tree [Internet]. 2017 [cited 2017 Jul 21]. [Full Text]

15. Hong W, Dong L, Huang Q, Wu W, Wu J, Wang Y. Prediction of severe acute pancreatitis using classification and regression tree analysis. Dig Dis Sci [Internet]. 2011 Dec [cited 2016 Feb 29];56(12):3664-71. [PubMed]

16. Huang W, Windsor JA. Fulminant or Early Severe Acute Pancreatitis Is Overlooked by Classifications of Severity. Crit Care Med [Internet]. 2017 Jul [cited 2017 Jul 22];45(7):e744-5. [PubMed]

17. Thomson A. Call for Subcategory of Severe Acute Pancreatitis: "Fulminant Acute Pancreatitis". Crit Care Med [Internet]. 2017 Feb [cited 2017 Jul 22];45(2):e2412. [Full Text] 